\title{
Article \\ Characterization of Soybean Protein Isolate-Food Polyphenol Interaction via Virtual Screening and Experimental Studies
}

\author{
Le Ao ${ }^{1,2,3,4}$, Panhang Liu 1,2,3, Annan Wu 1,2,3, Jing Zhao ${ }^{1,2,3, *}$ and Xiaosong Hu 1,2,3 \\ 1 College of Food Science and Nutritional Engineering, China Agricultural University, Beijing 100083, China; \\ a.kamio@163.com (L.A.); panhangliu@163.com (P.L.); s20213061005@cau.edu.cn (A.W.); \\ huxiaos@263.net (X.H.) \\ 2 China National Engineering Research Center for Fruit and Vegetable Processing, Beijing 100083, China \\ 3 Key Laboratory of Fruit and Vegetable Processing, Ministry of Agriculture, Beijing 100083, China \\ 4 China Academy of Machinery Science and Technology Group Co., Ltd., Beijing 100083, China \\ * Correspondence: zhaojing_cau@hotmail.com
}

Citation: Ao, L.; Liu, P.; Wu, A.; Zhao, J.; Hu, X. Characterization of Soybean Protein Isolate-Food Polyphenol Interaction via Virtual Screening and Experimental Studies. Foods 2021, 10, 2813. https:/ / doi.org/ $10.3390 /$ foods 10112813

Academic Editors: Marcello Iriti and Sara Vitalini

Received: 13 October 2021

Accepted: 12 November 2021

Published: 16 November 2021

Publisher's Note: MDPI stays neutral with regard to jurisdictional claims in published maps and institutional affiliations.

Copyright: (C) 2021 by the authors Licensee MDPI, Basel, Switzerland. This article is an open access article distributed under the terms and conditions of the Creative Commons Attribution (CC BY) license (https:/ / creativecommons.org/licenses/by/ $4.0 /)$.

\begin{abstract}
Background: Protein-polyphenol interactions have been widely studied regarding their influence on the properties of both protein and the ligands. As an important protein material in the food industry, soybean protein isolate (SPI) experiences interesting changes through polyphenols binding. (2) Methods: In this study, a molecular docking and virtual screening method was established to evaluate the SPI-polyphenol interaction. A compound library composed of 33 commonly found food source polyphenols was used in virtual screening. The binding capacity of top-ranking polyphenols (rutin, procyanidin, cyanidin chloride, quercetin) was validated and compared by fluorescence assays. (3) Results: Four out of five top-ranking polyphenols in virtual screening were flavonoids, while phenolic acids exhibit low binding capacity. Hydrogen bonding and hydrophobic interactions were found to be dominant interactions involved in soybean proteinpolyphenol binding. Cyanidin chloride exhibited the highest apparent binding constant (Ka), which was followed by quercetin, procyanidin, and rutin. Unlike others, procyanidin addition perturbed a red shift of SPI fluorescence, indicating a slight conformational change of SPI. (4) Conclusions: These results suggest that the pattern of SPI-polyphenol interaction is highly dependent on the detailed structure of polyphenols, which have important implications in uncovering the binding mechanism of SPI-polyphenol interaction.
\end{abstract}

Keywords: soybean protein isolate; polyphenol; molecular docking; fluorescence

\section{Introduction}

Protein-polyphenol interaction has been attracting more and more attention due to its beneficial influence to the properties of both the protein and polyphenols. Soybean protein isolate (SPI) is one of the most important protein products prepared from soybean. With the development of emerging plant-based foods, there are considerable interest in expanding the utilization of plant proteins because of their potential benefits in terms of sustainability, health, and ethical issues. However, some natural properties of SPI cannot meet the commercial needs, limiting the application of SPI in food processing. Recently, many studies indicate that the solubility, emulsifiability, thermal stability, and functional properties of SPI are altered when SPI is complexed with small molecules, especially polyphenol compounds [1,2]. EGCG binds SPI and enhances the foaming and emulsifying activity of SPI [3]. The forming of a rutin-SPI complex significantly increased the foaming capacity of SPI [4]. The underlying mechanisms include the exposure of hydrophobic amino acid residues, the change of protein secondary structure, the bridging of proteinsolvent interactions, etc. In another aspect, protein-polyphenol binding was also reported to modify the property of polyphenol itself, stabilizing the compound and increasing its bioavailability and bioactive functions such as antioxidant activity [5]. 
More and more polyphenol compounds are being tested for their protein binding capacities, while rare research had been carried out on the comparison of the behavior of different polyphenols. Dietary polyphenols exhibit many functional activities, with distinct structures varying with different categories and sources [6-10]. It is mainly composed of five categories, including flavonoids, phenolic acids, stilbenes, lignans and their derivatives, among which flavonoids are the most abundant [11]. Flavonoids are characterized by a unique $\mathrm{C} 3-\mathrm{C} 6-\mathrm{C} 3$ skeleton and further divided into six main subclasses including catechins, flavanols, flavones, flavanones, isoflavones, and anthocyanidins. Phenolic acids are featured by carboxylic acid groups, and stilbenes contain two benzene rings joined by a molecule of ethylene [12]. Lignans are phenolic dimers and oligomers possessing a 2,3-dibenzylbutane structure [13]. Distinct structures endow polyphenol compounds with miscellaneous physiochemical properties, likely affecting their protein-binding behavior. Thus, the characterization and comparison of protein-polyphenol interaction is important to understand how SPI interacts with different polyphenols, which has implications on the utilization of SPI-polyphenol complex in food industry.

In this study, 33 polyphenol compounds were selected from common food sources, including 25 flavonoids, 6 phenolic acids, 1 lignan, and 1 stilbenes, and compared regarding their SPI-binding capacity. The SPI-polyphenol interactions were investigated by molecular docking, virtual screening, and fluorescence quenching assays. Our work offers references for uncovering the SPI-polyphenol binding mechanisms and the utilization of polyphenols in SPI modification.

\section{Materials and Methods}

\subsection{Materials}

Soy protein isolate $(90.0 \%$ protein) used in this study was purchased from Shandong Biological Products Co., Ltd. (Linyi, China). Rutin, lignan, procyanidin, cyanidin chloride, and quercetin (purity $>90 \%$ ) were purchased from Solarbio Life Science Ltd. (Beijing, China). All other chemicals and regents were of analytical grade. Syringe filters were produced from Sigma-Aldrich (St. Louis, MO, USA).

\subsection{Binding Pockets Identification by Schrödinger Suites}

Schrödinger suites [14,15] (Schrödinger Release 2021-3: SiteMap, Schrödinger, LLC, New York, NY, USA, 2021) was used to predict the binding pockets on soybean 11S glycinin. The crystal structure of 11S glycinin (PDB ID: 1OD5) was prepared using the Protein Preparation Wizard in Maestro. Hydrogens were added, and the bond orders and formal charges were assigned. The hydrogen bond network of the protein was optimized, including the reorientation of hydroxyl and thiol groups and the prediction of the protonation states of aspartic acid, glutamic acid, and histidine. A brief minimization was carried out after the H-bond network optimization. The SiteMap module of Schrödinger was used to identify potential binding sites following a standard protocol.

\subsection{EGCG Docking and Pocket Selection}

As a known SPI binder, epigallocatechin gallate (EGCG) was used as a model compound and docked into the pockets predicted by Schrödinger Sitemap. Schrödinger Glide was used in molecular docking operations. The grid files were generated by the Receptor Grid Generation module after importing the protein structure prepared. The docking of EGCG into pockets was evaluated by the ligand docking function. The docking scores were used to select the most appropriate binding pocket.

\subsection{Virtual Screening of Food Source Polyphenols}

A virtual screening was carried out to evaluate the binding capacity of 33 commonly used polyphenols from different food sources (including rutin, procyanidin, quercetin, resveratrol, gallic acid, etc., see Supplemental Table S1) to SPI. The screening was performed using Schrödinger Glide based on the grid files generated in Section 2.3. The selected 
polyphenols were docked into the optimal pocket and evaluated by the ligand docking function. The molecular conformation change deviation was investigated by calculating the root mean square deviation (RMSD) of each atom. RMSD values of less than 2 were considered as successful in reproducing the protein-ligand interaction.

\subsection{Validation of SPI-Polyphenol Binding by Fluorescence Assays}

The intrinsic fluorescence spectra were measured using an F-7000 spectrofluorometer (HITACHI, Tokyo, Japan) at $25^{\circ} \mathrm{C}$ with a cell of $1 \mathrm{~cm}$ path length. The fluorescence was measured by exciting the protein at $280 \mathrm{~nm}$ and recording its emission spectra in the wavelength range of $310-450 \mathrm{~nm}$. The final protein concentration was kept at $0.135 \mathrm{mg} / \mathrm{mL}$. A series of aliquots of polyphenol solution $(0.1 \mathrm{mM})$ were sequentially added to $4 \mathrm{~mL}$ of SPI solution $(0.135 \mathrm{mg} / \mathrm{mL})$.

The fluorescence data were calculated using the Stern-Volmer equation:

$$
F_{0} / F=1+K_{s v}[Q] .
$$

In this equation, $F$ and $F_{0}$ are the fluorescence intensity of the protein solution with polyphenols and without, respectively, $K_{s v}$ is the quenching constant, and $[Q]$ is the concentration of polyphenols.

Binding parameters can be estimated using the quenching data by equation:

$$
\log \left[\left(F_{0}-F\right) / F\right]=\log K_{a}+n \log [Q] .
$$

In this equation, $K_{a}$ is the apparent binding constant, and $n$ is the number of binding sites.

\subsection{Statistical Analysis}

The statistical analysis software GraphPad (San Diego, CA, USA) was used to analyze the data. A comparison of the means was ascertained by Duncan's test at a $5 \%$ level of significance using one-way analysis of variance (ANOVA).

\section{Results and Discussion}

\subsection{Binding-Pocket Identification and EGCG Docking}

SPI is mainly composed of glycinin (11S), $\beta$-conglycinin (7S), and lipophilic proteins $[16,17]$. 11S glycinin has been used to construct hollow microcapsule as a delivery system for bioactive compounds [18]. Using a Schrödinger Sitemap, four binding pockets were obtained on soybean $11 \mathrm{~S}$ glycinin. To evaluate the accessibility of four pockets, molecular docking was carried out using epigallocatechin gallate (EGCG), a known SPI binder [3,19], as the ligand. As shown in Figure 1, EGCG was docked into the four pockets individually. Pocket 1 has the lowest binding energy, which is $-9.663 \mathrm{kcal} / \mathrm{mol}$, followed by Pocket $3(-6.828 \mathrm{kcal} / \mathrm{mol})$, Pocket $2(-5.575 \mathrm{kcal} / \mathrm{mol})$, and Pocket $4(-5.414 \mathrm{kcal} / \mathrm{mol})$. For Pocket 1, direct interactions were found between the phenol groups of EGCG, especially ring B and ring D, and amino acids of 11S. Glu172, His173, Val162, and Gly202 are highly involved in the interaction. Two hydroxyl groups on the B ring interact with Val162 and Gly202, separately. Two hydroxyl groups on the D ring interact with Glu172 and His173, separately. Thus, pocket 1 was chosen as the binding region in the following assays.

\subsection{Virtual Screening of Food Polyphenols against Soybean Protein}

A total of 33 commonly used polyphenols from different food sources were selected, including rutin, procyanidin, quercetin, resveratrol, gallic acid, etc. (Supplemental Table S1). A virtual screening was carried out to evaluate the binding capacity of different food polyphenols. As shown in Supplemental Table S1, rutin exhibited the lowest binding energy, which is $-7.969 \mathrm{kcal} / \mathrm{mol}$, followed by lignan $(-7.694 \mathrm{kcal} / \mathrm{mol})$ and procyanidin $(-7.526 \mathrm{kcal} / \mathrm{mol})$. The stereo view of the selected 11S-polyphenol complex is shown in Figure 2. Detailed interactions are shown in Figure 3. Both hydrogen bonding and hydrophobic interaction are likely involved in the rutin-11S complex $[3,20]$. A substantial 
amount of hydrogen bondings could form between $11 \mathrm{~S}$ amino acid side-chain protons and ring oxygens of rutin (Figure $3 \mathrm{~A}$ ), which likely contribute to its binding to SPI. The major amino acids involved in rutin binding include Glu172, His 173, Thr176, Arg 161, Glu200, and Gly202. For lignan, amino acids including Asp157, Arg161, Phe163, Thr176, Glu172, and Gly202 dominate the binding (Figure 3B). For procyanidin (Figure 3C), Val162, Tyr164, Thr176, Glu200, and Gly202 are highly involved. Less interactions were found for cyanidin chloride (Figure 3D) and quercetin (Figure 3E), with cyanidin chloride binding to Arg161, Glu172, Thr176, and quercetin binding to Arg161, Val162, Glu172, His173, and Met177.

A
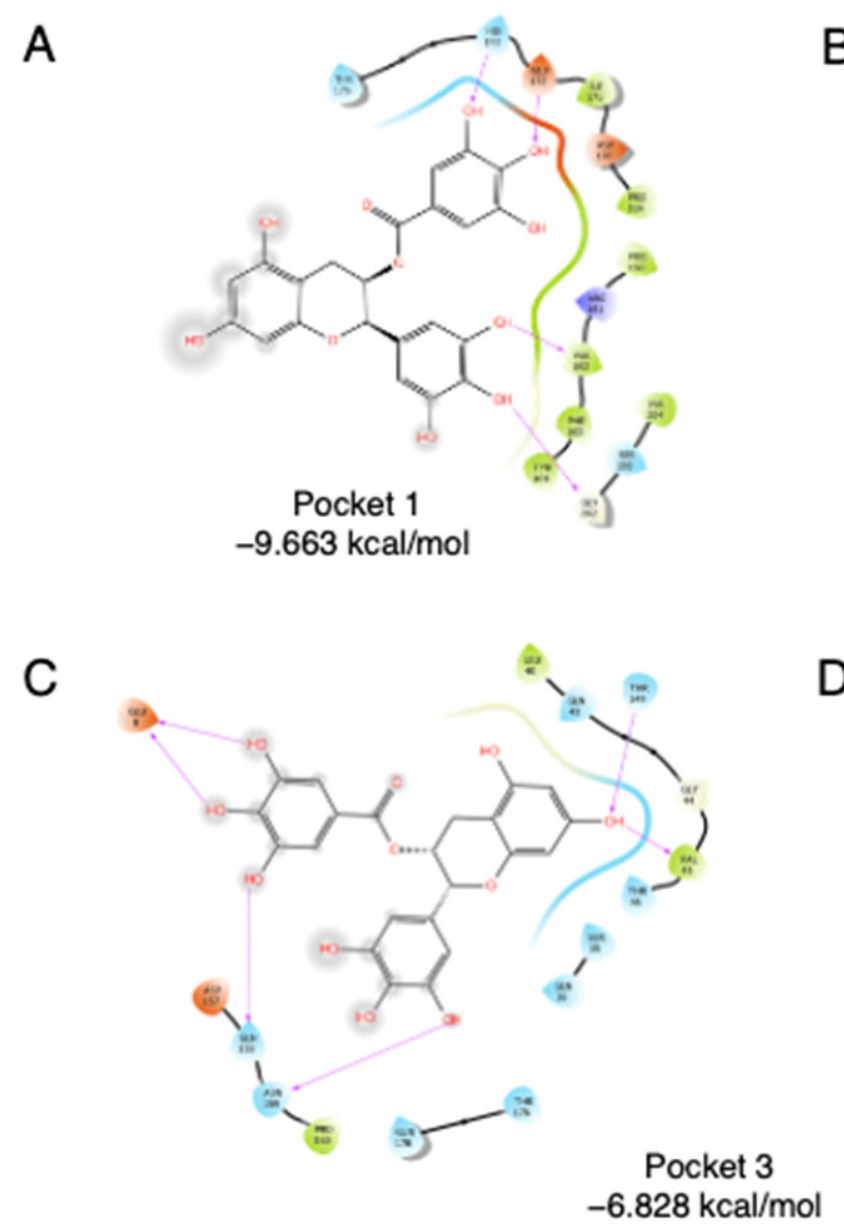

B

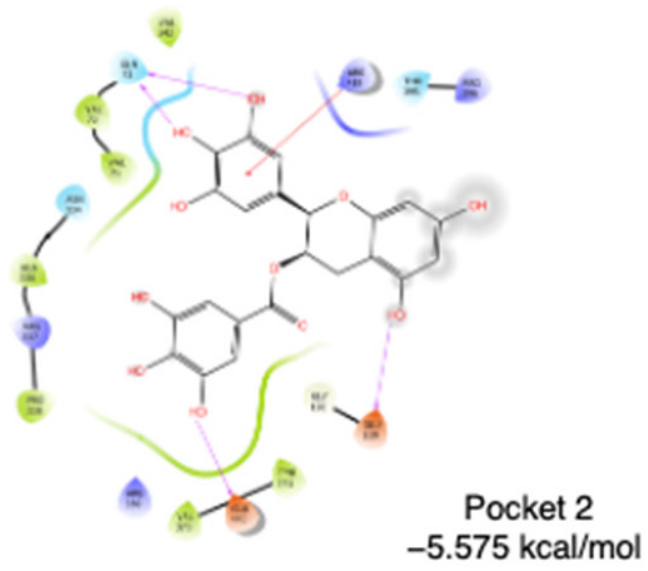

D

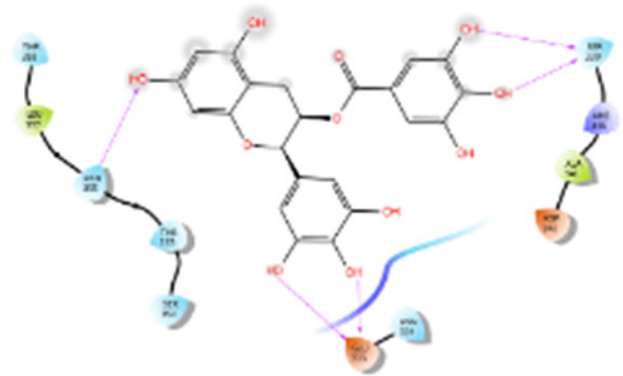

Pocket 4

$-5.414 \mathrm{kcal} / \mathrm{mol}$

Figure 1. Docking of EGCG into four pockets on soybean 11S globulin. (A) Pocket 1, (B) Pocket 2, (C) Pocket3, (D) Pocket4.

\subsection{Validation of Polyphenol-SPI Interaction by Fluorescence Quenching}

Fluorescence quenching assay has been widely used to characterize protein-ligand interactions [2,21]. Aromatic amino acids (mainly tryptophan and tyrosine) in protein can be excited by a laser wavelength around 280 and emitted a strong fluorescence signal, a.k.a. the intrinsic protein fluorescence [22]. Quencher molecules decrease the intrinsic fluorescence of protein in certain patterns. Polyphenol compounds have been reported to be fluorescence quenchers, and thus, the protein-polyphenol interaction can be detected by fluorescence assay [23]. In this study, the five top-ranking compounds from virtual screening, rutin, lignan, procyanidin, cyanidin chloride, and quercetin, were applied in fluorescence quenching assay to validate binding). 
A

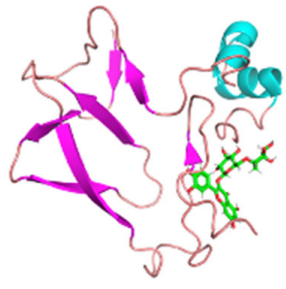

Rutin

$-7.969 \mathrm{kcal} / \mathrm{mol}$

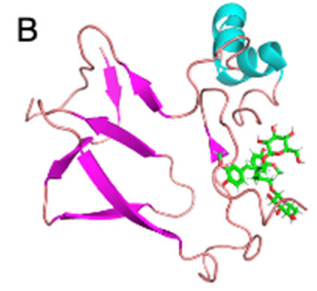

Lignan

$-7.694 \mathrm{kcal} / \mathrm{mol}$
C

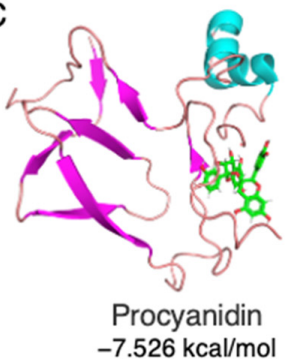

D

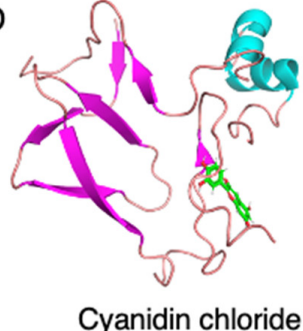

Cyanidin chloride

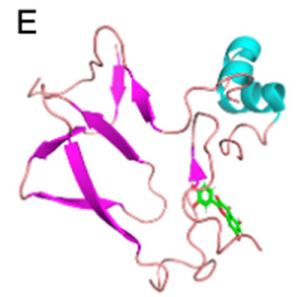

Quercetin $-6.803 \mathrm{kcal} / \mathrm{mol}$

Figure 2. The stereo view of top-ranking polyphenols complexed with soybean $11 \mathrm{~S}$ globulin. (A) Rutin, (B) lignan, (C) procyanidin, (D) cyanidin chloride, and (E) quercetin.

A

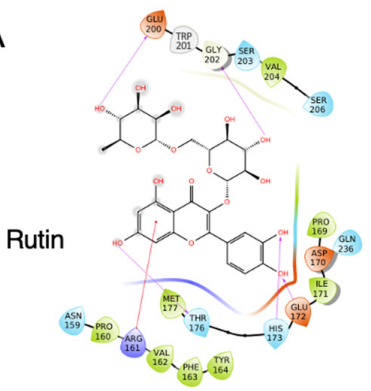

B

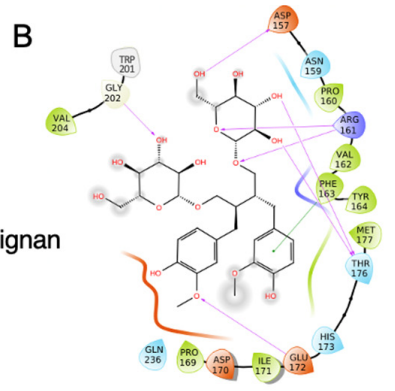

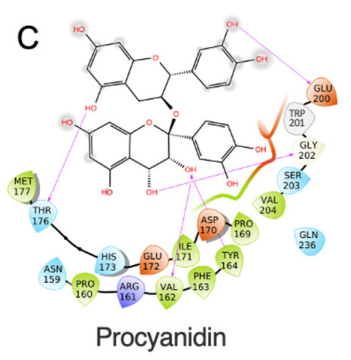
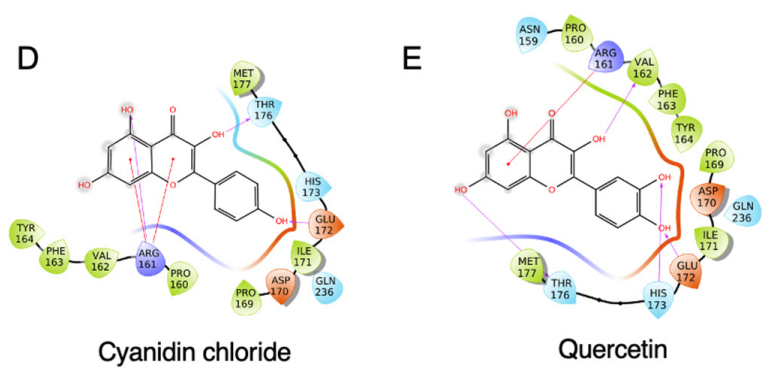

Figure 3. Interaction diagram of top-ranking polyphenols complexed with soybean 11S globulin. (A) Rutin, (B) lignan, (C) procyanidin, (D) cyanidin chloride, and (E) quercetin.

Rutin (quercetin-3-O-rutinoside) is a glycoside flavonoid abundantly found in many plants, such as buckwheat, apples, and tea [24]. It is reported to have antioxidant, antiinflammation, anti-tumor, and neuroprotective activities [25-28]. Rutin exhibited the lowest binding energy $(-7.969 \mathrm{kcal} / \mathrm{mol})$ in molecular docking. Figure 4 shows the fluorescence emission spectra of SPI with the addition of rutin. With the increase in rutin concentration (from 1 to $100 \mu \mathrm{M}$ ), the fluorescence intensity of SPI decreased progressively, confirming the interaction between rutin and SPI. As shown in Figure 4B (top), $F_{0} / F$ was fitted well against rutin concentration by the Stern-Volmer equation (Equation (1)). A linear Stern-Volmer plot was observed, which means that only one type of quenching mechanism occurs (dynamic or static). The quenching constant $K_{s v}$ was fitted to be $(0.039 \pm 0.0009) \times 10^{6} \mathrm{~L} \cdot \mathrm{mol}^{-1}$ (Table 1$)$. Binding parameters, the apparent binding constant $K_{a}$, and the average number of binding sites $n$ were obtained by fitting experi- 
mental data to Equation (2). It turns out that the rutin-SPI interaction exhibited a $K_{a}$ of $(0.007 \pm 0.001) \times 10^{6} \mathrm{~L} \cdot \mathrm{mol}^{-1}$ and an average number of binding sites as $1.40 \pm 0.034$.

A

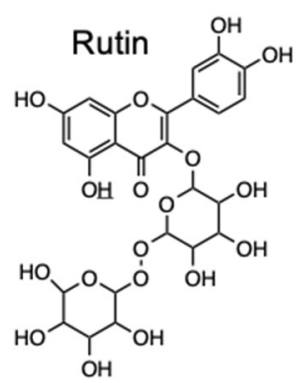

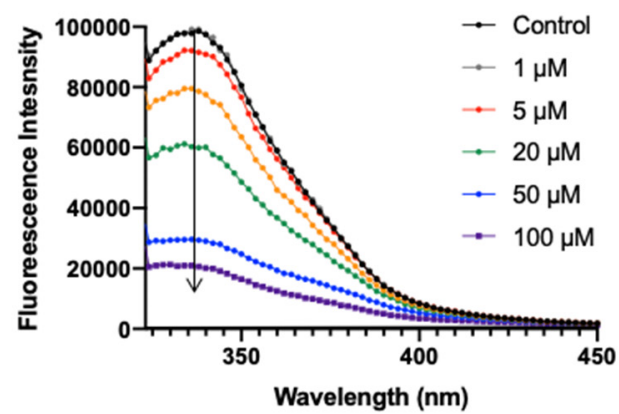

B
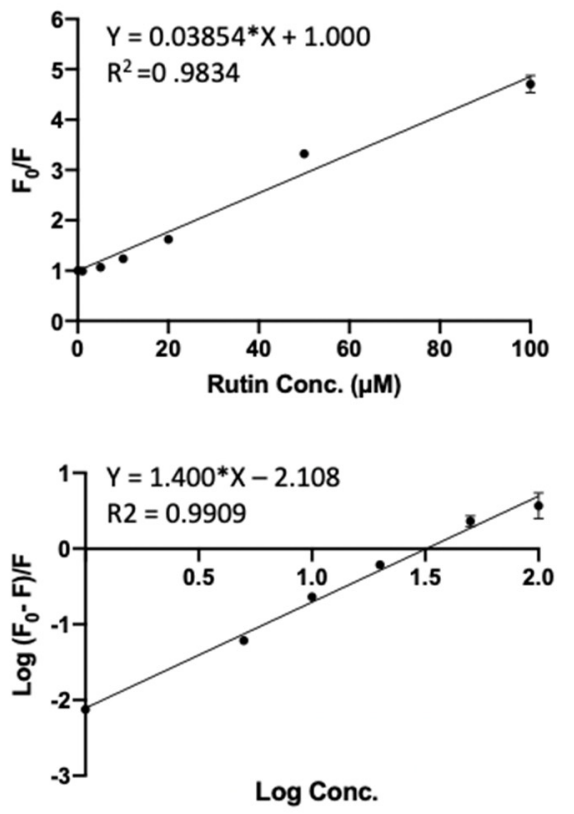

Figure 4. Fluorescence assay of SPI-rutin interaction. (A) The fluorescence emission profiles of SPI in the presence of different concentrations of rutin. The inset corresponds to the molecular structure of rutin. (B) The Stern-Volmer plot and binding parameter estimation plot.

Table 1. Binding parameters of selected SPI-polyphenol interactions.

\begin{tabular}{|c|c|c|c|}
\hline Number & $\begin{array}{c}K_{S V} \\
\left(\times 10^{6} \mathrm{~L} \cdot \mathrm{mol}^{-1}\right)\end{array}$ & $n$ & $\begin{array}{c}K_{a} \\
\left(\times 10^{6} \mathrm{~L} \cdot \mathrm{mol}^{-1}\right)\end{array}$ \\
\hline rutin & $0.039 \pm 0.0009^{c}$ & $1.40 \pm 0.034^{\mathrm{a}}$ & $0.007 \pm 0.001^{\mathrm{d}}$ \\
\hline procyanidin & $0.033 \pm 0.0003^{d}$ & $1.09 \pm 0.011^{b}$ & $0.024 \pm 0.002^{c}$ \\
\hline cyanidin chloride & $0.053 \pm 0.0010^{a}$ & $0.73 \pm 0.064^{c}$ & $0.861 \pm 0.039^{a}$ \\
\hline quercetin & $0.044 \pm 0.0017^{b}$ & $1.08 \pm 0.060^{b}$ & $0.028 \pm 0.003^{b}$ \\
\hline
\end{tabular}

Procyanidin is the most common subgroup of proanthocyanidins, which is commonly found in fruits (especially berries), some cereals, and root vegetables (onions cabbages, and radishes). It is a dimer or oligomer composed of epicatechin units and their galloyl derivatives $[29,30]$. The procyanidin used in this study is a B-type dimer (B2) construct. Procyanidin B2 has been reported to have beneficial effects on cardiovascular diseases and aging [31,32], which was claimed to be a potent inhibitor of COVID-19 protease recently [33]. It has a docking energy of $-7.526 \mathrm{kcal} / \mathrm{mol}$ in virtual screening. Similar to rutin, adding procyanidin induced the decrease in intrinsic fluorescence intensity of SPI. Notably, a red shift (from 347 to $349 \mathrm{~nm}$ ) is observed with increasing procyanidin concentration (from 1 to $50 \mu \mathrm{M}$; see Figure 5A), suggesting that the fluorophores of SPI are located in a more hydrophilic environment in the presence of procyanidin [22], which indicates that procyanidin may induce slight conformational changes of SPI. As shown in Figure 5B and Table 1, the quenching constant $K_{s v}$ for procyanidin was fitted to be $(0.033 \pm 0.0003) \times 10^{6} \mathrm{~L} \cdot \mathrm{mol}^{-1}$. The apparent binding constant $K_{a}$ and the average number of binding sites $n$ were obtained to be $(0.024 \pm 0.002) \times 10^{6} \mathrm{~L} \cdot \mathrm{mol}^{-1}$ and $1.09 \pm 0.011$, respectively. 
A
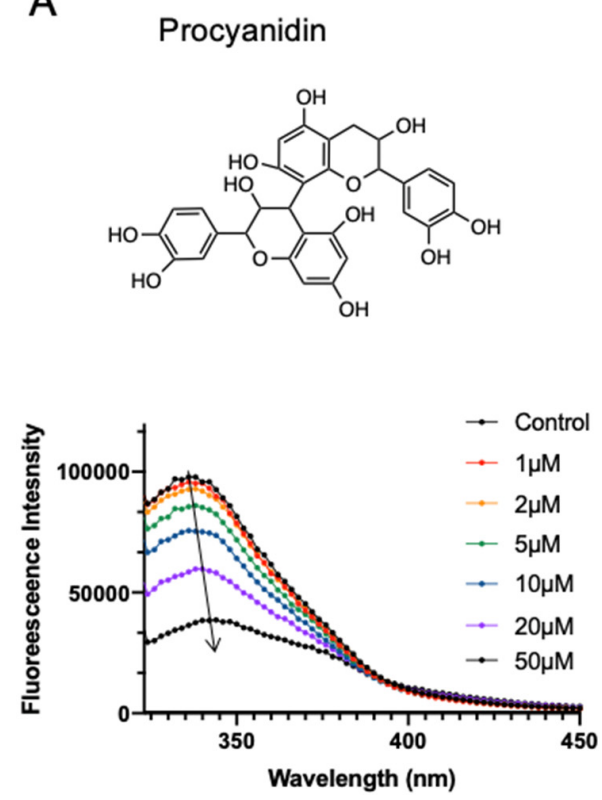

B $\quad{ }^{2.0} 7 \quad Y=0.03333^{*} X+1.000$
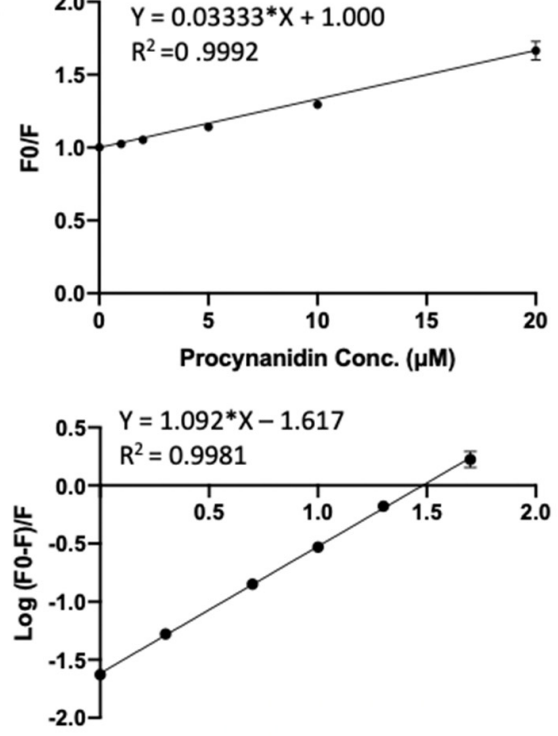

Log Conc.

Figure 5. Fluorescence assay of SPI-procyanidin interaction. (A) The fluorescence emission profiles of SPI in the presence of different concentrations of procyanidin. The inset corresponds to the molecular structure of procyanidin. (B) The Stern-Volmer plot and binding parameter estimation plot.

Figure 6 shows the SPI fluorescence quenching by cyanidin chloride, which has a docking energy of $-7.401 \mathrm{kcal} / \mathrm{mol}$ in virtual screening. Cyanidin chloride is a depolymerization product of procyanidin or naturally occurring compound that is found in berries, flowers, and vegetables. Compared to procyanidin, cyanidin chloride did not perturb the red shift of SPI fluorescence, indicating the existence of a different binding mode. A similar quenching constant $K_{s v}$ of $(0.053 \pm 0.0010) \times 10^{6} \mathrm{~L} \cdot \mathrm{mol}^{-1}$ was obtained (Table 1). Cyanidin has the highest apparent binding constant $K_{a}$ of $(0.861 \pm 0.039) \times 10^{6} \mathrm{~L} \cdot \mathrm{mol}^{-1}$ and the average number of binding sites $n$ fitted to be $0.73 \pm 0.064$.

A

\section{Cyanidin chloride}<smiles>Oc1ccc(-c2ccc(O)cc2O)c(O)c1</smiles>

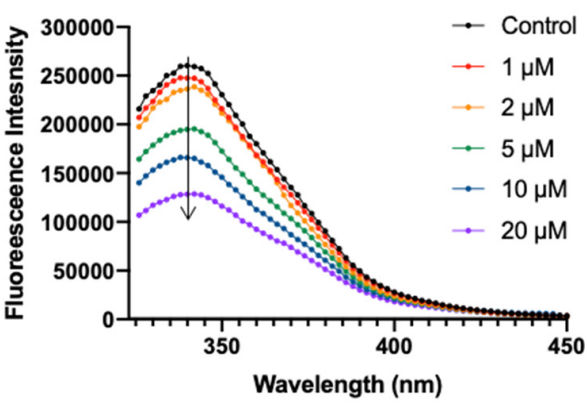

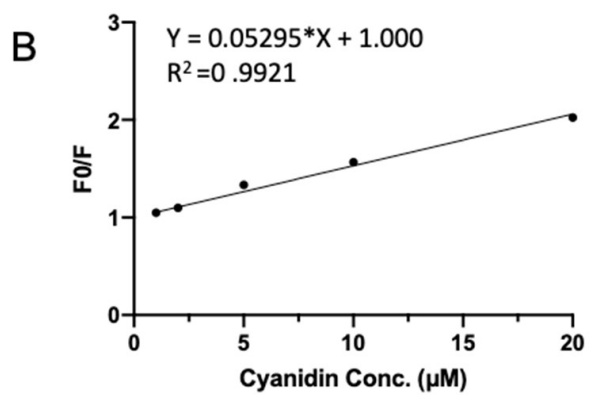

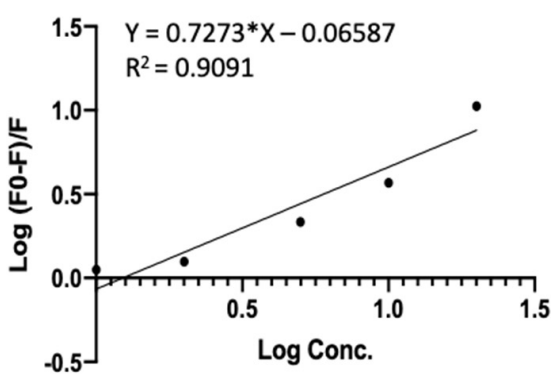

Figure 6. Fluorescence assay of SPI-cyanidin chloride interaction. (A) The fluorescence emission profiles of SPI in the presence of different concentrations of cyanidin chloride. The inset corresponds to the molecular structure of cyanidin chloride. (B) The Stern-Volmer plot and binding parameter estimation plot. 
Quercetin widely exists in our daily diet and has a wide range of biological functions including anti-inflammatory, antiviral, and anti-carcinogenic activities, as well as attenuating lipid peroxidation and platelet aggregation [34]. In addition to natural occurrence, it can also be released in the gastrointestinal tract from hydrolysis after oral administration of rutin. As shown in Figure 7A, quercetin quenched the intrinsic fluorescent of SPI in a concentration-dependent manner. The binding parameters fitted in Figure $7 \mathrm{~B}$ are shown in Table 1. Quercetin exhibited a similar $K_{s v}$ of $(0.044 \pm 0.0017) \times 10^{6} \mathrm{~L} \cdot \mathrm{mol}^{-1}$ as the other compounds. The apparent binding constant $K_{a}$ and the average number of binding sites $n$ were fitted to be $(0.028 \pm 0.003) \times 10^{6} \mathrm{~L} \cdot \mathrm{mol}^{-1}$ and $1.08 \pm 0.060$, respectively.

A<smiles>O=C(O)C1Oc2cc(O)cc(O)c2OC1c1ccc(O)c(O)c1</smiles>

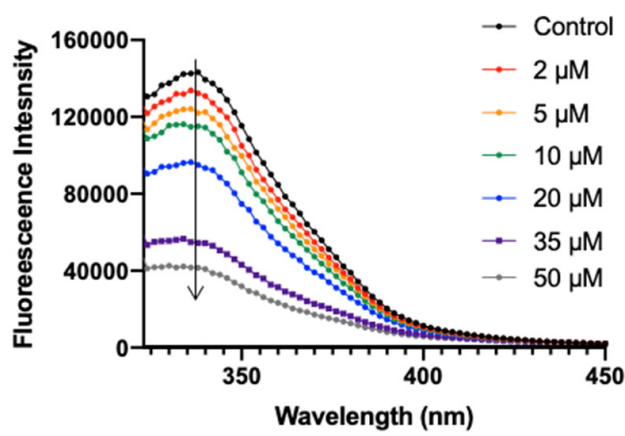

B
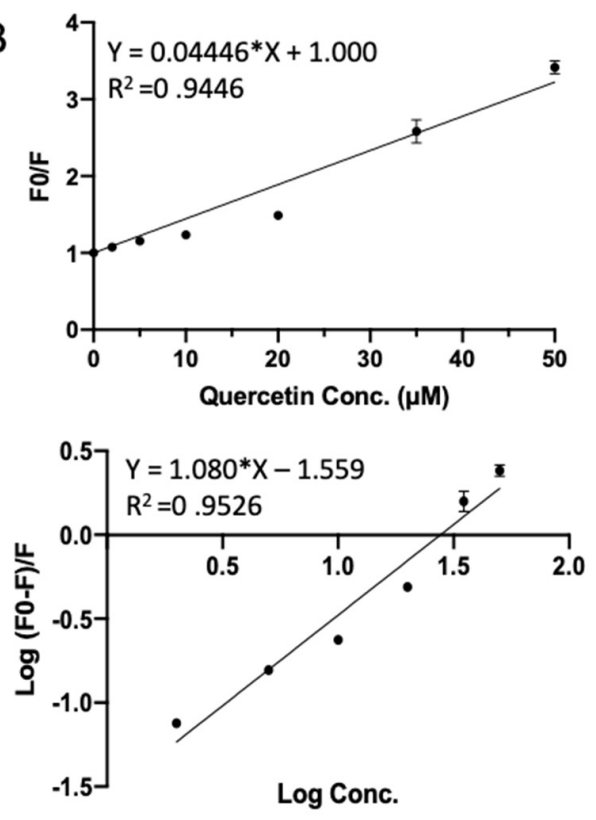

Figure 7. Fluorescence assay of SPI-quercetin interaction. (A) The fluorescence emission profiles of SPI in the presence of different concentrations of quercetin. The inset corresponds to the molecular structure of quercetin. (B) The Stern-Volmer plot and binding parameter estimation plot.

\section{Conclusions}

Numerous polyphenol compounds had been reported regarding their protein binding capacities, while rare research had been carried out on the comparison of the behavior of different polyphenols. In this study, 33 dietary polyphenol compounds including 25 flavonoids, 6 phenolic acids, 1 lignan, and 1 stilbene were analyzed and compared regarding their SPI binding capacity. Molecular docking, virtual screening, and fluorescence quenching assays were performed. Interestingly, four out of five top-ranking polyphenols in virtual screening were flavonoids, while phenolic acids exhibit relatively low binding capacity. It suggests that the SPI-polyphenol interaction is highly dependent on the variety of polyphenols. In vitro SPI binding of top-ranking polyphenols (rutin, procyanidin, cyanidin chloride, quercetin) was tested and compared by fluorescence quenching assays. Cyanidin chloride exhibited the highest apparent binding constant $K_{a}$, while procyanidin perturbed a red shift of SPI fluorescence, indicating a slight conformational change of SPI. These results indicate that polyphenols with a different structure may exhibit distinct protein binding patterns. Our work provides a combined strategy of molecular docking, virtual screening, and in vitro validation assays in charactering the SPI-polyphenol interactions, which has important implications in uncovering the SPI-polyphenol binding mechanism on a molecular level and offers a fundamental basis for the utilization of the SPI-polyphenol complex in food systems. 
Supplementary Materials: The following are available online at https:/ / www.mdpi.com/article/10 .3390/foods10112813/s1, Table S1: The docking scores of 33 commonly found food polyphenols.

Author Contributions: Conceptualization, J.Z. and X.H.; methodology, J.Z.; software, L.A.; formal analysis, L.A., P.L. and A.W.; investigation, L.A., P.L. and A.W.; writing-original draft preparation, L.A.; writing-review and editing, J.Z.; funding acquisition, J.Z. All authors have read and agreed to the published version of the manuscript.

Funding: This research received no external funding.

Institutional Review Board Statement: Not applicable.

Data Availability Statement: Not applicable.

Conflicts of Interest: The authors declare no conflict of interest.

\section{References}

1. Djuardi, A.U.P.; Yuliana, N.D.; Ogawa, M.; Akazawa, T.; Suhartono, M.T. Emulsifying properties and antioxidant activity of soy protein isolate conjugated with tea polyphenol extracts. J. Food Sci. Technol. 2020, 57, 3591-3600. [CrossRef]

2. Chen, G.; Wang, S.; Feng, B.; Jiang, B.; Miao, M. Interaction between soybean protein and tea polyphenols under high pressure. Food Chem. 2019, 277, 632-638. [CrossRef]

3. You, Y.; Yang, L.; Chen, H.; Xiong, L.; Yang, F. Effects of (-)-Epigallocatechin-3-gallate on the functional and structural properties of soybean protein isolate. J. Agric. Food Chem. 2021, 69, 2306-2315. [CrossRef]

4. Ye, J.; Deng, L.; Wang, Y.; McClements, D.J.; Luo, S.; Liu, C. Impact of rutin on the foaming properties of soybean protein: Formation and characterization of flavonoid-protein complexes. Food Chem. 2021, 362, 130238. [CrossRef] [PubMed]

5. Frazier, R.A.; Papadopoulou, A.; Mueller-Harvey, I.; Kissoon, D.; Green, R.J. Probing protein-tannin interactions by isothermal titration microcalorimetry. J. Agric. Food Chem. 2003, 51, 5189-5195. [CrossRef]

6. Manach, C.; Scalbert, A.; Morand, C.; Rémésy, C.; Jiménez, L. Polyphenols: Food sources and bioavailability. Am. J. Clin. Nutr. 2004, 79, 727-747. [CrossRef] [PubMed]

7. Fraga, C.G.; Croft, K.D.; Kennedy, D.O.; Tomás-Barberán, F.A. The effects of polyphenols and other bioactives on human health. Food Funct. 2019, 10, 514-528. [CrossRef] [PubMed]

8. Xia, E.Q.; Deng, G.F.; Guo, Y.J.; Li, H. Bin Biological activities of polyphenols from grapes. Int. J. Mol. Sci. 2010, 11, 622-646. [CrossRef]

9. Chen, G.L.; Mutie, F.M.; Xu, Y.B.; Saleri, F.D.; Hu, G.W.; Guo, M.Q. Antioxidant, anti-inflammatory activities and polyphenol profile of rhamnus prinoides. Pharmaceuticals 2020, 13, 55. [CrossRef]

10. Coppo, E.; Marchese, A. Antibacterial Activity of Polyphenols. Curr. Pharm. Biotechnol. 2014, 15, 380-390. [CrossRef]

11. Singla, R.K.; Dubey, A.K.; Garg, A.; Sharma, R.K.; Fiorino, M.; Ameen, S.M.; Haddad, M.A.; Al-Hiary, M. Natural Polyphenols: Chemical Classification, Definition of Classes, Subcategories, and Structures. J. AOAC Int. 2019, 102, 1397-1400. [CrossRef] [PubMed]

12. Moreno, J.; Peinado, R. Enological Chemistry; Elsevier: Amsterdam, The Netherlands, 2012.

13. Deshpande, S.D. Encyclopedia of Food Sciences and Nutrition; Academic Press: Cambridge, MA, USA, 2003 ; pp. 619-627.

14. Halgren, T.A. Identifying and characterizing binding sites and assessing druggability. J. Chem. Inf. Model. 2009, 49, 377-389. [CrossRef]

15. Halgren, T. New method for fast and accurate binding-site identification and analysis. Chem. Biol. Drug Des. 2007, 69, 146-148. [CrossRef] [PubMed]

16. Ma, W.; Xie, F.; Zhang, S.; Wang, H.; Hu, M.; Sun, Y.; Zhong, M.; Zhu, J.; Qi, B.; Li, Y. Characterizing the structural and functional properties of soybean protein extracted from full-fat soybean flakes after low-temperature dry extrusion. Molecules 2018, $23,3265$. [CrossRef] [PubMed]

17. Din, J.U.; Sarwar, A.; Li, Y.; Aziz, T.; Hussain, F.; Shah, S.M.M.; Yi, G.; Liu, X. Separation of Storage Proteins (7S and 11S) from Soybean Seed, Meals and Protein Isolate Using an Optimized Method Via Comparison of Yield and Purity. Protein J. 2021, 40, 396-405. [CrossRef] [PubMed]

18. Zhao, H.; Guo, M.; Ding, T.; Ye, X.; Liu, D. Exploring the mechanism of hollow microcapsule formation by self-assembly of soy 11s protein upon heating. Food Hydrocoll. 2020, 108, 105379. [CrossRef]

19. Yan, S.; Xu, J.; Zhang, X.; Xie, F.; Zhang, S.; Jiang, L.; Qi, B.; Li, Y. Effect of pH-shifting treatment on the structural and functional properties of soybean protein isolate and its interactions with (-)-epigallocatechin-3-gallate. Process Biochem. 2021, 101, 190-198. [CrossRef]

20. Dai, T.; Chen, J.; McClements, D.J.; Hu, P.; Ye, X.; Liu, C.; Li, T. Protein-polyphenol interactions enhance the antioxidant capacity of phenolics: Analysis of rice glutelin-procyanidin dimer interactions. Food Funct. 2019, 10, 765-774. [CrossRef] [PubMed]

21. Li, X.; Wang, S. Study on the interaction of (+)-catechin with human serum albumin using isothermal titration calorimetry and spectroscopic techniques. New J. Chem. 2015, 39, 386-395. [CrossRef]

22. Lakowicz, J.R. Principles of Fluorescence Spectroscopy; Springer Science \& Business Media: New York, NY, USA, 2006 ; ISBN 0387312781. 
23. El-Messery, T.M.; Mwafy, E.A.; Mostafa, A.M.; El-Din, H.M.F.; Mwafy, A.; Amarowicz, R.; Ozçelik, B. Spectroscopic studies of the interaction between isolated polyphenols from coffee and the milk proteins. Surf. Interfaces 2020, 20, 100558. [CrossRef]

24. Chackalamannil, S.; Rotella, D.P.; Ward, S.E. Comprehensive Medicinal Chemistry III; Elsevier: Amsterdam, The Netherlands, 2017; ISBN 9780128032008.

25. Enogieru, A.B.; Haylett, W.; Hiss, D.C.; Bardien, S.; Ekpo, O.E. Rutin as a potent antioxidant: Implications for neurodegenerative disorders. Oxid. Med. Cell. Longev. 2018, 2018, 6241017. [CrossRef]

26. Liu, S.; Adewole, D.; Yu, L.; Sid, V.; Wang, B.; Karmin, O.; Yang, C. Rutin attenuates inflammatory responses induced by lipopolysaccharide in an in vitro mouse muscle cell (C2C12) model. Poult. Sci. 2019, 98, 2756-2764. [CrossRef]

27. Moutinho, M.S.S.; Aragão, S.; Carmo, D.; Casaca, F.; Silva, S.; Ribeiro, J.; Sousa, H.; Pires, I.; Queiroga, F.; Colaço, B.; et al. Curcumin and rutin down-regulate cyclooxygenase-2 and reduce tumor-associated inflammation in HPV16-transgenic mice. Anticancer Res. 2018, 38, 1461-1466. [CrossRef] [PubMed]

28. Song, H.L.; Zhang, X.; Wang, W.Z.; Liu, R.H.; Zhao, K.; Liu, M.Y.; Gong, W.M.; Ning, B. Neuroprotective mechanisms of rutin for spinal cord injury through anti-oxidation and anti-inflammation and inhibition of p38 mitogen activated protein kinase pathway. Neural Regen. Res. 2018, 13, 128-134. [CrossRef] [PubMed]

29. Gali, H.U.; Perchellet, E.M.; Gao, X.M.; Karchesy, J.J.; Perchellet, J.P. Comparison of the inhibitory effects of monomeric, dimeric, and trimeric procyanidins on the biochemical markers of skin tumor promotion in mouse epidermis in vivo. Planta Med. 1994, 60, 235-239. [CrossRef]

30. Appeldoorn, M.M.; Vincken, J.P.; Gruppen, H.; Hollman, P.C.H. Procyanidin dimers A1, A2, and B2 are absorbed without conjugation or methylation from the small intestine of rats. J. Nutr. 2009, 139, 1469-1473. [CrossRef]

31. Novakovic, A.; Marinko, M.; Jankovic, G.; Stojanovic, I.; Milojevic, P.; Nenezic, D.; Kanjuh, V.; Yang, Q.; He, G.W. Endotheliumdependent vasorelaxant effect of procyanidin B2 on human internal mammary artery. Eur. J. Pharmacol. 2017, 807, 75-81. [CrossRef] [PubMed]

32. Xiao, Y.; Dong, J.; Yin, Z.; Wu, Q.; Zhou, Y.; Zhou, X. Procyanidin B2 protects against D-galactose-induced mimetic aging in mice: Metabolites and microbiome analysis. Food Chem. Toxicol. 2018, 119, 141-149. [CrossRef]

33. Ravi, L.; Rex, C.M.; Sreenivas, A.B.K. Procyanidin B2 of Cassia fistula a Potent Inhibitor of COVID-19 Protease: A Molecular Dynamic Simulation Analysis. Asian J. Pharm. 2020, 14, 274-281.

34. Li, Y.; Yao, J.; Han, C.; Yang, J.; Chaudhry, M.T.; Wang, S.; Liu, H.; Yin, Y. Quercetin, inflammation and immunity. Nutrients 2016, 8,167 . [CrossRef] 